\title{
An Algorithm for Constructing 3D Struts
}

\author{
George W. Hart \\ Department of Computer Science, Stony Brook University, Stony Brook, NY 11794, U.S.A. \\ E-mail: george@georgehart.com
}

Received July 22, 2008; revised December 2, 2008.

\begin{abstract}
A simple robust "strut algorithm" is presented which, when given a graph embedded in 3D space, thickens its edges into solid struts. Various applications, crystallographic and sculptural, are shown in which smooth high-genus forms are the output. A toolbox of algorithmic techniques allow for a variety of novel, visually engaging forms that express a mathematical aesthetic. In sculptural examples, hyperbolic tessellations in the Poincaré plane are transformed in several ways to three-dimensional networks of edges embodied within a plausibly organic organization. By the use of different transformations and adjustable parameters in the algorithms, a variety of attractive forms result. The techniques produce watertight boundary representations that can be built with solid freeform fabrication equipment. The final physical output satisfies the "coolness criterion," that passers by will pick them up and say "Wow, that's cool!"
\end{abstract}

Keywords sculpture, solid modeling, echinoderm, algorithms, fine arts, computer-aided design

\section{Introduction}

As a sculptor, I am interested in creating physical objects that are novel, natural, strongly self-coherent, and convey a sense of "structural inevitability." In my work, I use a variety of mathematical ideas and physical materials ${ }^{[1]}$. The motivation for the work presented below is to realize visions for structures that are similar to forms in the echinoderm family, but happen not to exist in nature. The algorithms are used to produce boundary representations that are physically realized via solid freeform fabrication techniques. Accordingly, the generating algorithms must be robust enough to produce "watertight" triangulated boundary representations consisting of one connected manifold.

Two basic ideas are repeatedly employed below. First, hyperbolic tessellations in the Poincaré plane are transformed (in various ways) to three-dimensional networks of edges. Then these edge networks are thickened to solid struts with a simple robust "strut algorithm". To illustrate its versatility, the strut algorithm is also applied to produce interesting crystallographic models. A variety of high-genus forms result, due to the use of different transformations and a number of adjustable parameters in the algorithms.

\section{Strut Algorithm}

The input is a graph embedded in 3D space, i.e., $(x, y, z)$ coordinates have been assigned to each vertex. We understand each edge as a line segment connecting the incident vertices and we assume the embedding does not intersect itself anywhere. There are many ways to transform an embedded graph into a $3 \mathrm{D}$ form. One idea is to create a small sphere around each vertex, create a cylinder around each edge, and take the 3D Boolean union of these components. If the spheres and cylinders are not too large, there will be no intersections, so the surface is a manifold. But this approach faces a familiar practical problem that 3D Boolean unions can be numerically difficult when intersecting boundary representations with floating point vertex coordinates. Given thousands of spheres and cylinders to combine, some unions will fail with currently available commercial software, and a watertight boundary representation is unlikely to result. More fundamentally, the resulting "ball and stick" structure gives a visual impression of a chemical or mathematical model - a different aesthetic than I am after, and a single connected surface is required for the final smoothing operations.

The 3D forms presented here all involve an algorithm that generates a high-genus surface that wraps around all the edges, as a ball-and-stick model does, but requires no Boolean unions and has a more controllable geometry. It is similar to the algorithm described in [2] but it is more adaptable and experiments show it is more robust. Some of the examples below resulted in invalid meshes (having intersecting polygons) using

Regular Paper

Portions of this material appeared in Proceedings of IEEE Solid Modeling International'08. 
the older algorithm, which led to this new approach. The algorithm is presented in the context of particular sculptural forms, but it applies to any sets of segments embedded in general position in three-space. Although simple and straightforward, I have not seen a similar algorithm described elsewhere, so it is worth documenting, as converting from edges to solid struts is a frequently requested operation. Experiments with a wide range of segment topologies, angles, and lengths indicate the algorithm is very robust.

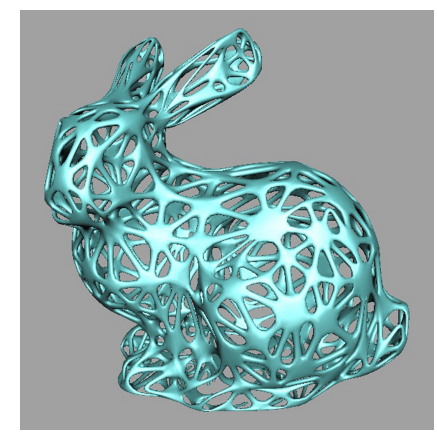

Fig.1. Output of strut algorithm for 500-triangle bunny model.

Fig.1 provides an illustrative example. The Stanford bunny model was simplified to 500 triangles, and then the edges of the triangles were used as input to the strut algorithm. The output is a watertight set of triangles which is the boundary of a solid network of connected struts that wraps around the bunny edges. The procedure is simple and fast enough that it could be made available as a standard operator in 3D design software (e.g., Maya, Rhinoceros, or AutoCAD) for creating interesting solid forms.

The geometric input to the algorithm is a list of vertices, given as $(x, y, z)$ coordinates, and a list of index pairs indicating which vertices are joined by edges.
Then for each end of each edge, the vertices of a regular polygon that encircles the edge are created. For each polygon, the radius, number of vertices, rotational "phase," and the position along the edge are controllable parameters, as described below. Fig.2(a) indicates the polygons as triangles around the four edges that meet at one vertex. Taking the convex hull of these vertices gives facets of a "ball" wrapping the vertex, shown in Fig.2(b). The faces normal to the edges are dropped, so there is a hole for each of the original polygons. Then for each edge, take the convex hull of the polygons at the two ends of the edge. Again, dropping the facets normal to the edge leaves facets of a "strut" wrapping the edge shown in Fig.2(c). Finally, combine all these facets - from every vertex and every edge into one set to obtain a complete boundary representation. The algorithm is fast because it mainly consists of small convex hull operations and orthogonality tests. The result is watertight because the same polygon vertices are used both for vertex wrapping and for edge wrapping.

Using triangles, as illustrated in Fig.2, gives a low face count for a high-genus object. In the examples below, $n$-gons are used, with $6 \leqslant n \leqslant 12$, for rounder appearing struts. The distance from the polygons to the vertices is chosen to ensure the polygons do not overlap. The minimum angle between edges at a vertex is found, which determines the radius of a sphere on which the polygons are placed. Half the length of the shortest incident edge is the radius upper bound, guaranteeing adjacent balls do not overlap.

Fig.2(d) illustrates a fine point of the algorithm. When the convex hull of the polygons around each vertex is constructed, the vertex itself is also included as an input point. In most cases, this has no effect on the result, as the vertex is usually interior to the hull of the edge-surrounding polygons. However, if the edges all

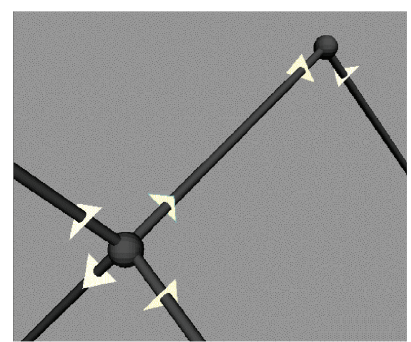

(a)

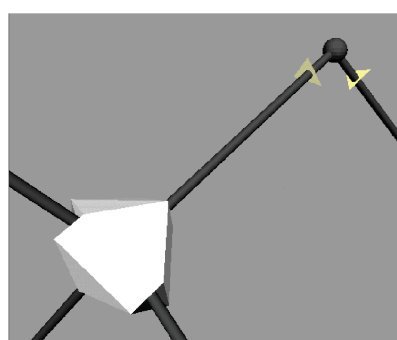

(b)

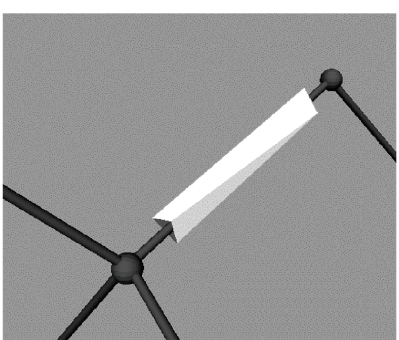

(c)

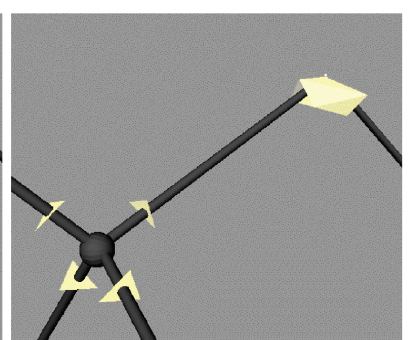

(d)

Fig.2. Strut algorithm. (a) A polygon is created around each end of each segment, illustrated here as triangles. (b) For each vertex, the convex hull of the nearby polygons and the vertex is constructed, but then the polygons themselves are removed from the hull. Typically the vertex is inside the hull and does not appear in the result. (c) For each edge, the convex hull of its two polygons is constructed, and again the two polygons are removed from the hull. (d) In Step (b), the vertex itself is sometimes part of the result, when the incident edges happen to lie in one hemisphere. 
lie in an acute cone, the center of the sphere is part of the output hull, and appears as the apex of the struts meeting at that vertex.

\section{Crystallographic Examples}

To demonstrate the versatility of the strut algorithm, we first apply it to some complex regular lattices which are very interesting to hold in the hand and study as physical models. Fig.3(a) shows a portion of the diamond crystal lattice in a standard ball and stick chemical model form. Each sphere represents a carbon atom, which bonds to four surrounding neighbors. Fig.3(b) shows a $4 \mathrm{~cm}$ plastic model of the same structure, generated by the strut algorithm, which made a smooth surface around the embedded edges. The model is made of nylon on a Selective Laser Sintering (SLS) machine.

One beautiful property of the diamond lattice is that there is space inside it for another copy of itself. Applying the strut algorithm to the "double diamond" lattice gives two independent intertwined networks, as seen in Fig.4(a). When produced by SLS, the two components of Fig.4(b) fit in the same 4cm cubic volume, and can be moved slightly, independently of each other. This

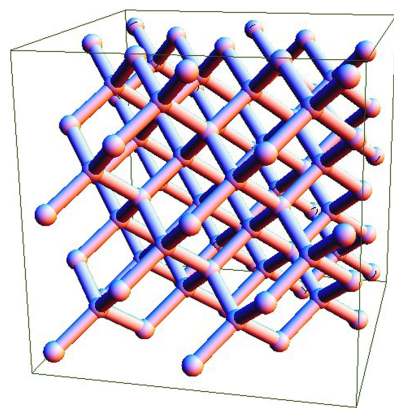

(a)

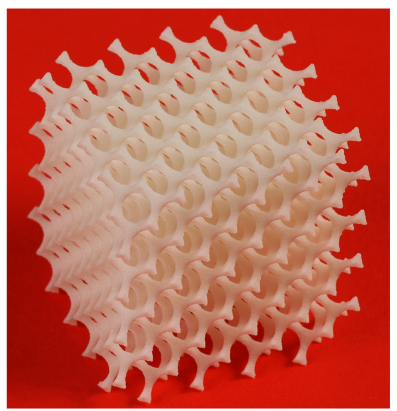

(b)

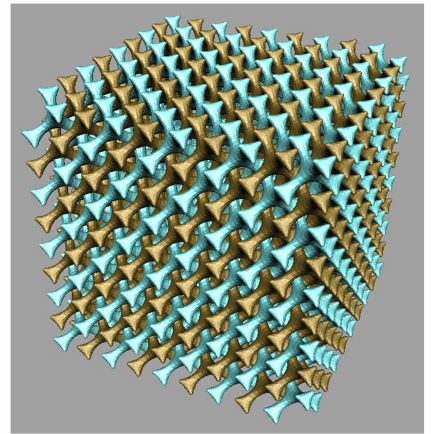

(a)

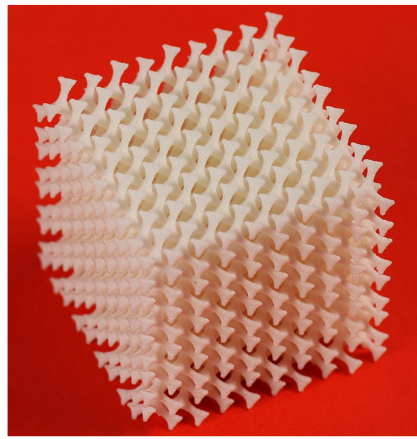

(b)

Fig.4. Double Diamond lattice. (a) Computer rendering. (b) $4 \mathrm{~cm}$ SLS model output of strut algorithm.

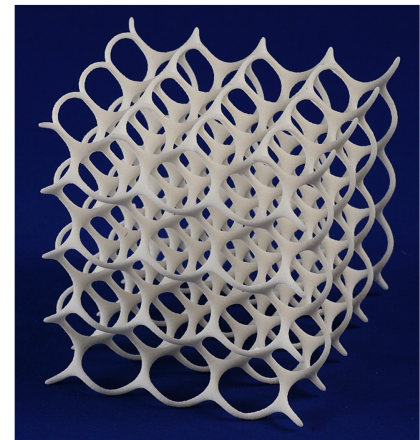

(a)

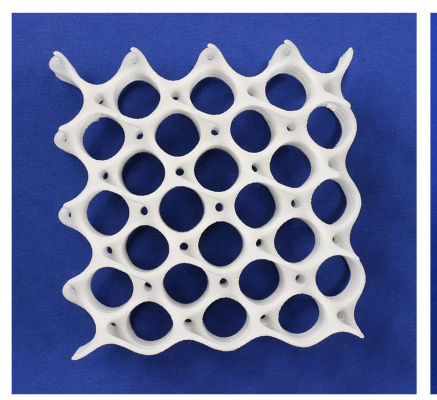

(c)

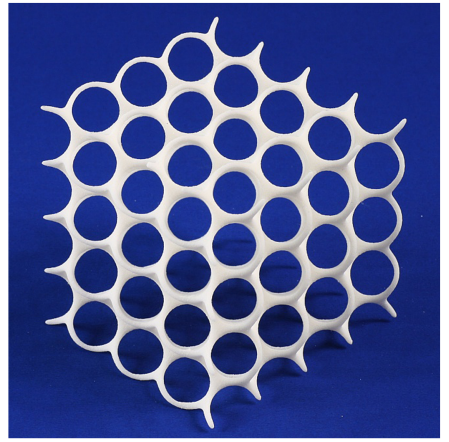

(b)

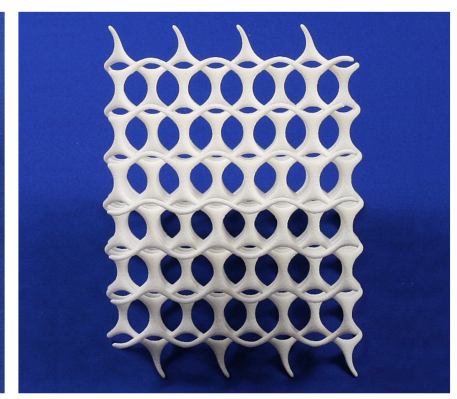

(d)

Fig.3. Diamond lattice. (a) Traditional ball and stick model. (b) $4 \mathrm{~cm}$ SLS model output of strut algorithm.

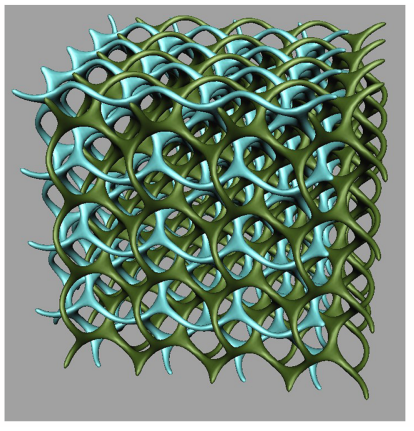

(a)

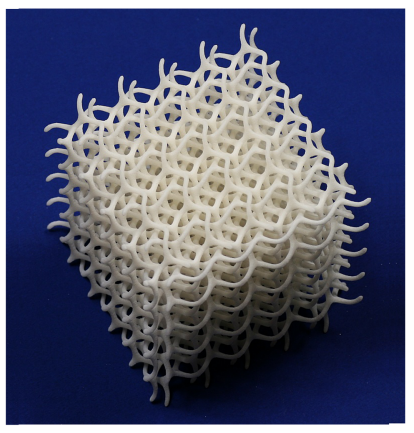

(b)

Fig.5. (10, 3)-a network. (a d) Four views of the 8cm SLS model output of strut algorithm.

Fig.6. Doubled $(10,3)$-a network. (a) Computer rendering. (b c) Two views of the $8 \mathrm{~cm}$ SLS model output of strut algorithm. 
structure occurs in a sodium-thallium alloy, where the sodium atoms take the positions of the carbons of one diamond lattice and the thallium atoms take the positions of the carbons of the other diamond lattice ${ }^{[3]}$.

Another interesting lattice is the "(10, 3)-a" network shown in Fig. $5^{[4]}$. The history and geometry of this fascinating structure are summarized in [5]. Figs. $5(\mathrm{a}) \sim 5(\mathrm{~d})$ show four views of an $8 \mathrm{~cm}$ model of the structure, as generated by this strut algorithm. There are many different aspects to it when projected in different directions.

Most interestingly, Wells ${ }^{[4]}$ observed that the "(10, 3)-a" lattice can interpenetrate space with a copy of its mirror image. Fig.6(a) shows a rendering to clarify that there are two independent components. Figs. 6(b) and 6 (c) show two views of this immensely complex interlocked structure, as produced by the strut algorithm. Again, the two parts are free to wiggle slightly.

\section{Sculpture Examples}

The following examples all begin with a hyperbolic tessellation, map its edges in some way to segments embedded in three-space, use the strut algorithm to form a high-genus manifold boundary wrapping around the struts, and apply some sort of smoothing. They are motivated by an underwater aesthetic, but are not meant to imply that the techniques are limited to this.

We begin with some background on hyperbolic tessellations. Fig.7 shows several hyperbolic tessellations in the Poincaré disk ${ }^{[6,7]}$. The first is the $\{7,7,7\}$ tessellation, which means that at each vertex three regular heptagons meet. As the sum of the angles around each vertex is greater than 360 , this tessellation cannot be constructed in the Euclidean plane. However, it is straightforwardly constructed with regular heptagons in the hyperbolic plane and then can be mapped to the Euclidean unit disk in various ways. In Fig.7(a), the center of a polygon is the center of the disk, while for Fig.7(b) a translation in the hyperbolic plane was applied, so the identical hyperbolic tessellation is mapped to the disk in a way that displays a vertex at the center.

Each hyperbolic tessellation in Fig.7 is "vertex uniform", which means each is specifiable by the cyclic configuration of regular polygons around each vertex. In all the examples presented, the Poincaré mapping is used to transform from the hyperbolic plane to the unit disk in Euclidean space. This mapping transforms geodesic lines in hyperbolic space to circular arcs orthogonal to the edges of the disk. Note that tessellations may be chiral, e.g., Fig.7(f). An infinite amount of complexity appears within epsilon of the circular

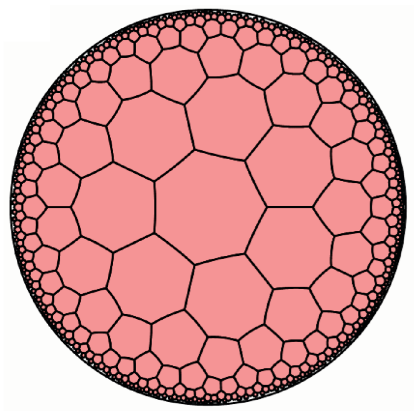

(a)

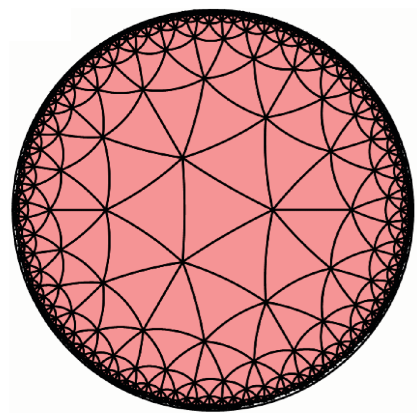

(d)

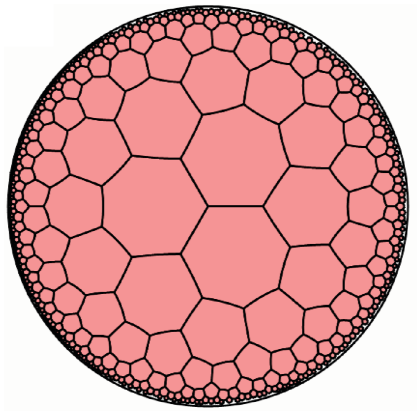

(b)

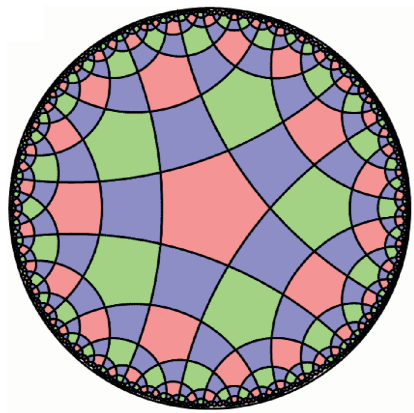

(e)

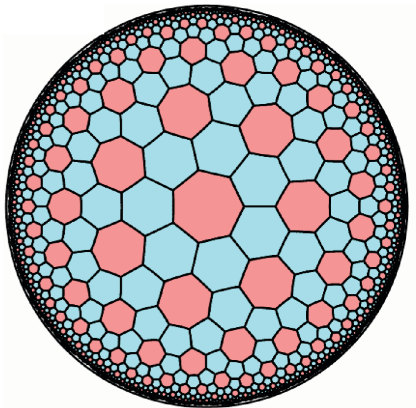

(c)

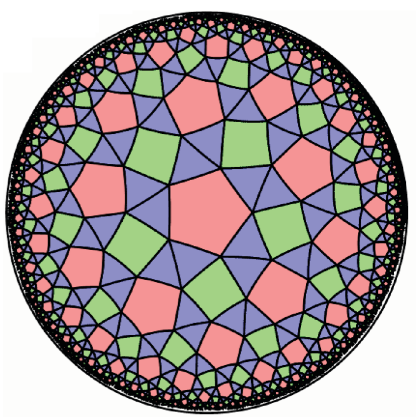

(f)

Fig.7. Some uniform hyperbolic tessellations in the Poincaré plane. (a) $\{7,7,7\}$. (b) $\{7,7,7\}$. (c) $\{7,6,6\}$. (d) $\{3,3,3,3,3,3,3\}$. (e) $\{5,4,5,4\}$. (f) $\{5,3,4,3,3\}$. 
boundary, so each tessellation is truncated to show only a selectable number of polygons around the center. Other mappings, particularly the Klein disk and upper half-plane model could be used in similar ways for creating three-dimensional forms in Euclidean space, but the Poincaré model was selected for its aesthetic properties.

By selecting a tessellation and a translation, we have available visually interesting geometric structures with various radial symmetries. Although there are only finitely many uniform tessellations in the Euclidean plane, there are infinitely many uniform tessellations in the hyperbolic plane. So one is not likely to quickly run out of interesting patterns. There are many varieties of nonuniform tessellations that may be considered in future work.

Several artists have used hyperbolic tessellations as a foundation for artistic design, most famously M. C. Escher ${ }^{[8]}$. Recent examples, usually two-dimensional, can be found in the artwork of Douglas Dunham, Heleman Ferguson, Craig Kaplan, Irene Rousseau, and Carlo Sequin, among others ${ }^{[9]}$. A series of novel approaches for adapting the edges of hyperbolic tessellations into three-dimensional forms are presented below.

\section{1 "Sand Dollars"}

Fig.8(a) shows the application of this algorithm to the tessellation of Fig.7(a). Note how the thickness of the disk tapers towards the edges. In the applications illustrated here, the edge-encircling polygons' radii are chosen at each vertex to be proportional to the average length of the edges meeting at that vertex. The struts then are thicker in proportion to their length, approximating a constant aspect ratio, but smoothly varying from the larger regions to the smaller regions, which is important in giving a sense of organic form. Fig.8(a) uses triangular struts, which give a low facet count and a more chiseled angular look, while Fig.8(b) shows foursided struts and the thickness parameter (polygon radius scaling factor) is increased. In Fig.8(c), 12-gons were used instead of triangles, so the struts appear effectively circular. Fig.8(d) shows the result using 6-gons, then applying standard polygon subdivision smoothing techniques in a post-processing step to soften the form. Smoothing is similarly applied in other examples below.

Fig.9 shows four examples of physical models made of nylon on a Selective Laser Sintering (SLS) machine. The flat white color and tapering perforated disk form is suggestive of a "sand dollar" but with a more interesting pattern of openings. Some of the smallest edge details in the geometry description file are too fine to be built given the resolution of the SLS machine. So

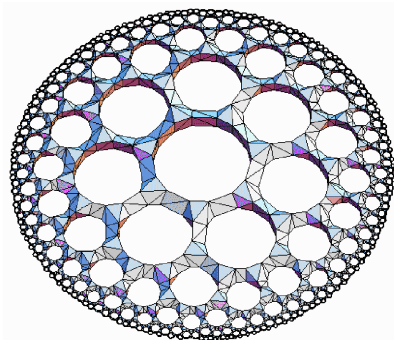

(a)

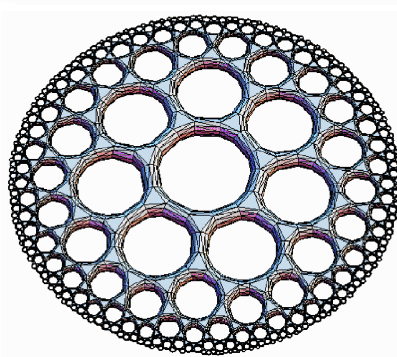

(c)

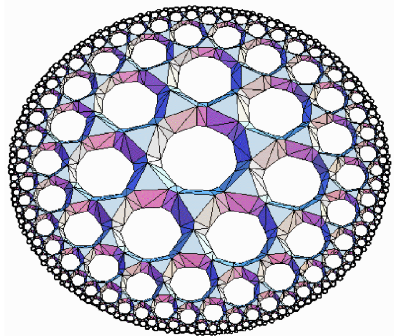

(b)

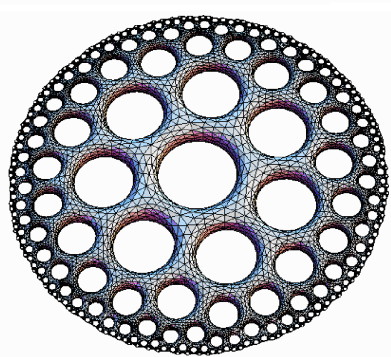

(d)
Fig.8. Visualizations of "Sand Dollars" based on $\{7,7,7\}$ tessellation. (a) Triangular struts. (b) Square struts and greater thickness. (c) 12-gon struts. (d) 6-gon struts with subdivision smoothing.

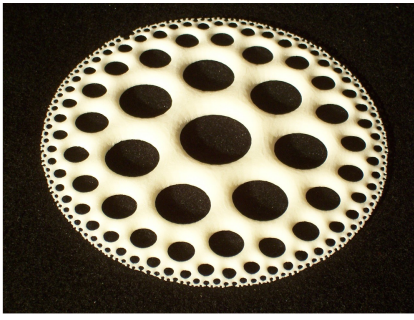

(a)

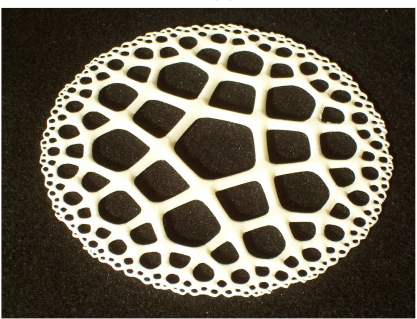

(c)

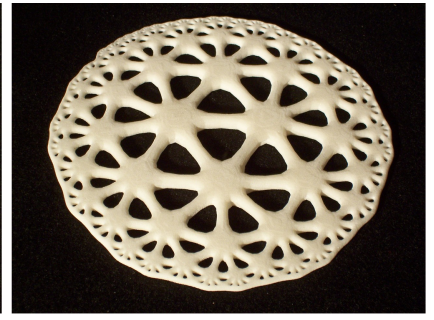

(b)

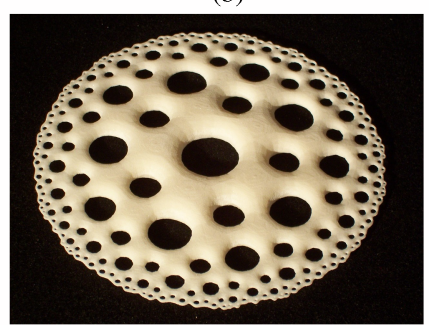

(d)
Fig.9. Sand Dollars, nylon, SLS, 6 inch diameter. (a) $\{7,7,7\}$. (b) $\{3,3,3,3,3,3,3\}$. (c) $\{5,4,5,4\}$ thin. (d) $\{5,4,5,4\}$ thick.

the edges are frayed in places, adding to their impression of being a natural artifact. Observe that Figs.9(c) and $9(\mathrm{~d})$ are based on the same $\{5,4,5,4\}$ tessellation but differ in the strut thickness, illustrating how the strut algorithm allows the designer to adjust between a linear and a bulbous visual impression. 


\subsection{Parabolic Polyp}

The "sand dollars" above are effectively sculptural, but almost two-dimensional. For a stronger 3D impact, the hyperbolic tessellation can be lifted out of the plane. Introducing a height function, $f(x, y)$ defined on the unit disc allows a variety of 3D designs. For the "sand dollars," each $(x, y)$ point of the Poincaré disk was mapped to a point $(x, y, 0)$ to embed the plane in $3 \mathrm{D}$. The next simplest idea is to use an arbitrary height function and map $(x, y)$ to $(x, y, f(x, y))$.

Fig.10(a) shows a design based on the vertexcentered $\{7,7,7\}$ tessellation of Fig.7(b), but with a height function that is parabolic in $r=\sqrt{\left(x^{2}+y^{2}\right)}$ : rising, reaching a maximum at radius $R$, then starting to descend. Specifically, the height was chosen to be proportional to $1-((r-R) / R)^{2}$, with $R=0.6$. As illustrated, triangular struts were used, so the form has an architectural feeling. It would make a dramatic design for a pavilion. Fig.10(b) shows a physical model made on an SLS machine.

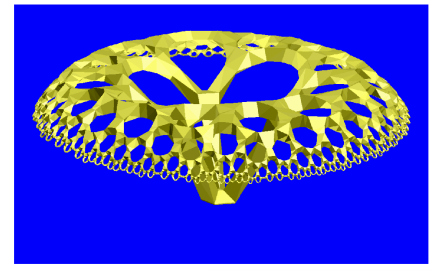

(a)

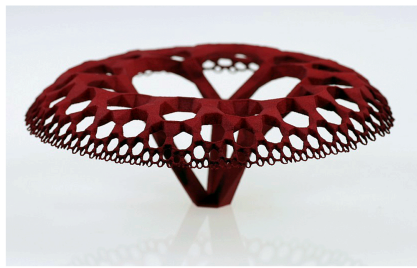

(b)
Fig.10. Parabolic Polyp. (a) Visualization. (b) Realization, nylon, SLS, 6-inch diameter.

\subsection{Helical Sweeps}

Considering the Poincaré disk in polar coordinates, $(r, \theta)$, we can sweep around it $k$ times, letting $\theta$ vary from 0 to $2 k \pi$. Choosing a height function linear in $\theta$, i.e., of the form $\alpha \theta$, results in a springy helix, as seen in Fig.11(a). Its projection to the $X Y$ plane is the same

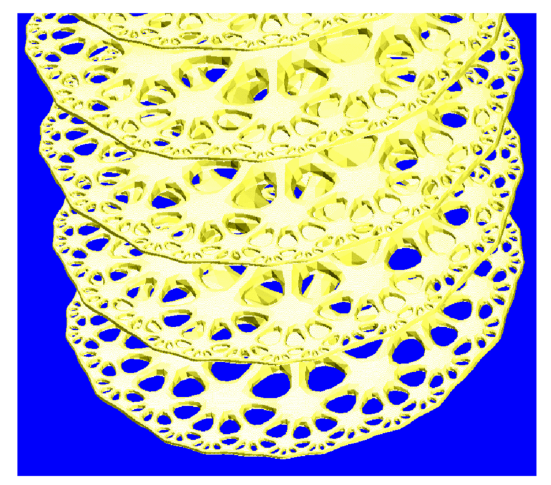

(a)

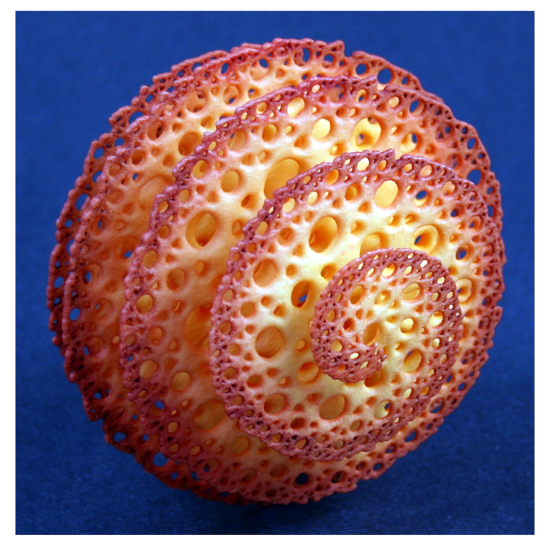

(c)

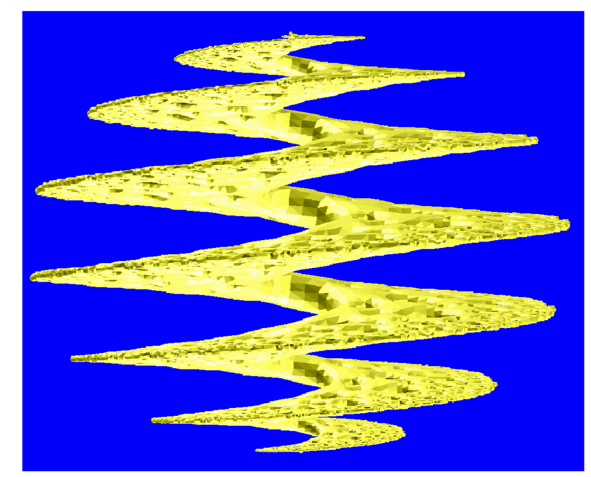

(b)

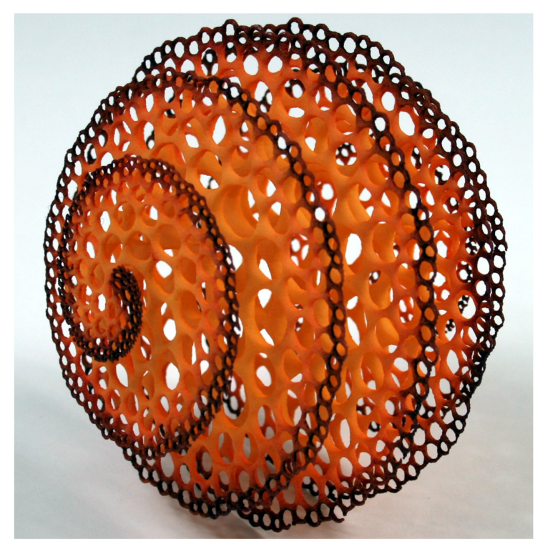

(d)

Fig.11. Helical forms. (a) Simple helix applied to $\{3,3,3,3,3,3,3\}$. (b) Side view of a helix proportioned to fit in a sphere. (c) $\{5$, $3,4,3,3\}$ realization, nylon, SLS, 4 -inch diameter. (d) $\{7,6,6\}$ realization, nylon, SLS, 4-inch diameter. 


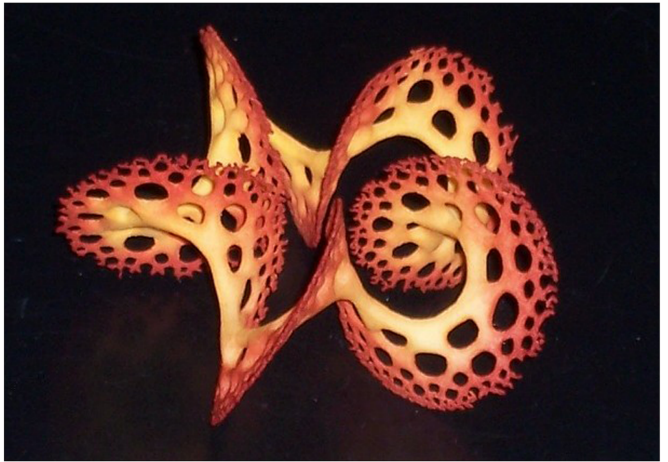

(a)

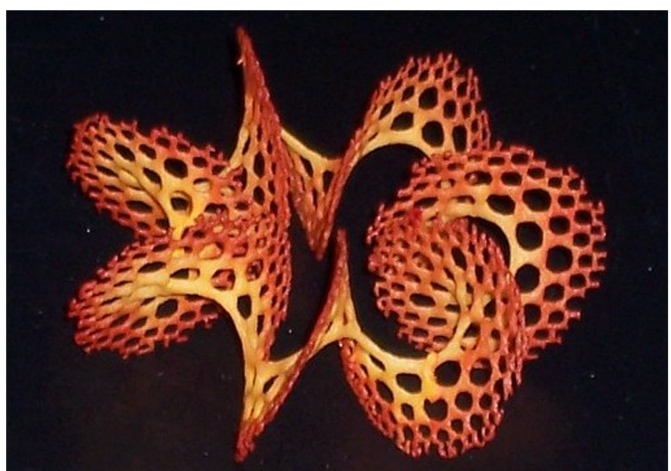

(b)

Fig.12. Toroidal forms, nylon, SLS, 3-inch diameter, hand dyed. (a) $\{5,4,5,4\}$. (b) $\{7,6,6\}$.

as that of Fig.9(b). This can be seen as a multiple covering of the Poincaré disk, tiled to be uniform at almost all vertices, but there is one unbounded polygon at the center with infinitely many sides.

For greater visual interest, such a helix could be scaled radially as well, e.g., to make nautilus-like conical forms, based on, e.g., an Archimedean or equiangular spiral. A different outer form was chosen here: the height is scaled as $\sin (\alpha \theta)$ and the radius is scaled as $\cos (\alpha \theta)$, so that the form nestles inside a sphere when $\theta$ varies from $-2 k \pi$ to $+2 k \pi$. Fig.11(b) shows a side view, to illustrate how it fits in a circle.

Figs. 11(c) and 11(d) show two sculptures based on this idea, using the $\{5,3,4,3,3\}$ and $\{7,6,6\}$ tessellations, respectively. They give a sense of being at home among the seaweed. I hand-dyed the nylon SLS models in Fig.6 with a radial color gradient. For this family of forms, a color gradient adds to the naturally organic impression and provides contrast and depth cues that make it easier to apprehend the $3 \mathrm{D}$ form from a $2 \mathrm{D}$ image.

\subsection{Toroidal Sweeps}

Extending the above idea, the sweep can be transformed to wrap around a point moving in a circle, creating a toroidal form with a spiral swirl. Mathematically, this is accomplished with standard rotating Frenet frame techniques parameterized by the sweep angle $\theta$. As an example, Fig.12 shows two such sculptures. The first example is based on the $\{5,4,5,4\}$ tessellation wrapping five times around a torus. The second is based on the $\{7,6,6\}$ tessellation wrapping seven times around a torus. These display a 5 -fold and a 7 -fold radial symmetry respectively, when viewed straight down from the top. Again, I hand-dyed them with a gradient color scheme as a simple way to artistically emphasize their organic nature. The results impress the viewer as simultaneously naturalistic yet alien.

\subsection{Knotted Sweep}

To produce a topologically intriguing object, the toroidal sweep can be replaced with a sweep with a frame rotating about a knotted path. Fig. 13 shows an unsmoothed and as-yet unrealized design for a knotted form with nine cycles based on the $\{7,6,6\}$ tessellation. Its sweep path is the simplest knotted path: a trefoil knot. The frequency and phase of the rotation was chosen so that the sculpture has a three-fold rotational symmetry axis. Repeated experimentation was necessary to find parameters which resulted in no selfintersections.

\subsection{Polyhedral Forms}

The final technique presented here is to take an angular wedge of the Poincaré disk and use it to build

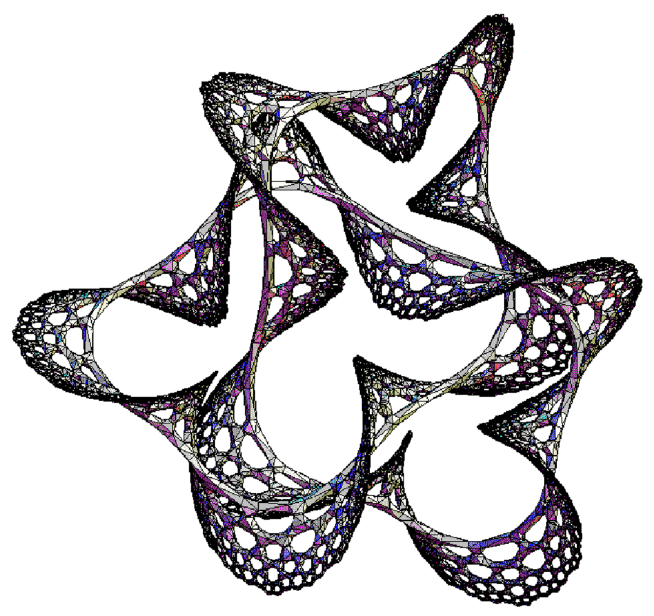

Fig.13. Knotted Kelp, visualization. 


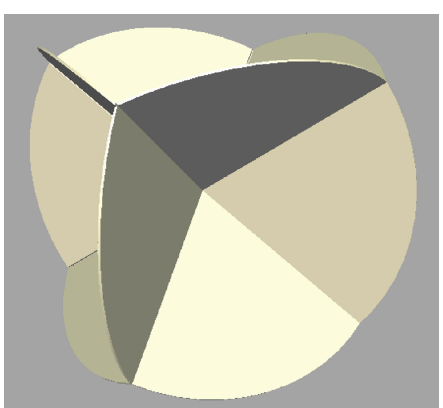

Fig.14. Cube's twelve edges replaced by circular sectors.

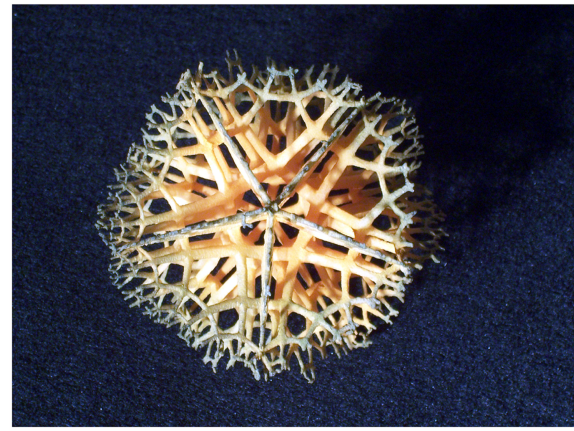

(a)

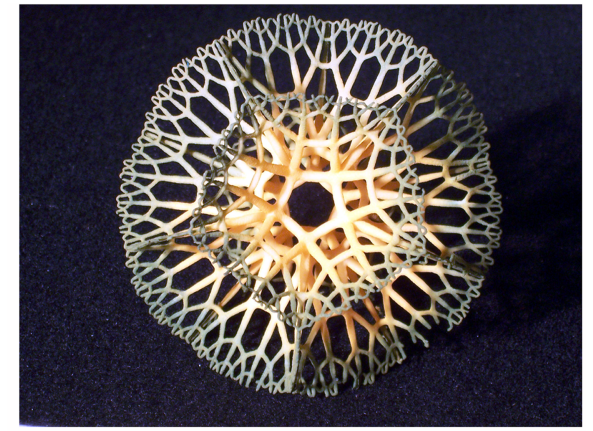

(b)

Fig.15. Polyhedral forms based on $\{5,4,5,4\}$, nylon, SLS, 4-inch diameter. (a) Icosahedral. (b) Dodecahedral.

spherical forms based on the edges of a polyhedron. A polyhedron edge subtends some angle when viewed from the center of the polyhedron. A circular segment of the same angle can fit into that angular space, as illustrated in Fig.14 for the twelve edges of a cube. In place of each circular wedge, we can use a sector of a tessellation in the Poincaré plane, to create an arrangement of segments. To maintain the polyhedral symmetry, the wedge in the Poincare plane should have mirror symmetry and be of size $2 \pi / n$ if the tessellation has $n$-fold symmetry. Note that the wedges we use and the original edges of the polyhedron need not span the identical central angle, because we can apply linear interpolation in $\theta$ as part of the mapping. So any size wedge of the Poincaré plane might be used, but to minimize distortion, one can select a symmetric wedge close in angle to the edge being replaced.

For a richer form, we use an icosahedron and dodecahedron instead of a cube in the examples presented here. Two sculptures, both based on a wedges of the $\{5,4,5,4\}$ tessellation, are shown in Fig.15. Again, a radial color gradient was hand applied. These delicate frameworks are in some ways reminiscent of Ernst Haeckel's drawings of microscopic life forms.

\section{Implementation}

The algorithms described above were coded using Mathematica, which provides a convenient library of primitive functions but is not a high-performance environment. As currently coded - for the author's experimental development rather than with efficient data structures - the more complex examples required several minutes to generate on a laptop PC. Careful recoding using a compiled language should reduce the execution time by a significant factor. A library of Mathematica functions for creating hyperbolic tessellations was used as the starting point ${ }^{[10]}$. Post-processing with
Maya was used for subdivision smoothing.

\section{Conclusion}

A robust toolbox with adjustable parameters is used to produce a variety of visually interesting $3 \mathrm{D}$ designs, suitable for building on solid freeform fabrication equipment. By wrapping surfaces around segments in a water-tight manner, high-genus sculptures are generated which have both a mathematical structure and an organic character. Algorithmically generated sculptural forms were presented which incorporate patterns based on tessellations of the hyperbolic plane. Assorted transformations are used to map the Poincaré disk to 3D objects. Their organic feel (and engineering strength) results in part from strut diameters that are proportional to length.

There is no objective correctness criterion for this type of design. The final sculptures are considered to be successful if they pass the "coolness test", that passers by stop and say "Wow, that's cool!" then pick them up and turn them about in their hands to view them from many angles. By that criterion, this work has been observed to be successful.

As a successful sculpture is difficult to capture from only one camera position, the images may not convey a complete sense of each form. The reader is invited to download the $3 \mathrm{D}$ models available at the website ${ }^{[11]}$ and explore them in a 3D viewer, or better, build them on a local fabrication machine.

Acknowledgment Thank Jim Quinn for the SLS production.

\section{References}

[1] Hart G W. The Geometric Aesthetic. Chapter 10, The Visual Mind II, Michele Emmer (ed.), MIT Press, 2005. 
[2] Hart G W. Solid-Segment Sculptures. Mathematics and Art, Claude Brute (ed.), Springer-Verlag, 2002.

[3] http://de.wikipedia.org/wiki/Eduard_Zintl.

[4] Wells A F. Three Dimensional Nets and Polyhedra. Wiley, 1977.

[5] Stephen T Hyde, Michael O'Keeffe, David M Proserpio. A short history of an elusive yet ubiquitous structure in chemistry, materials and mathematics. Angewandte Chemie, International Edition, 2008, 47(42): 7996-8000.

[6] Anderson James. Hyperbolic Geometry. Springer, 1999.

[7] Coxeter H S M. Non-Euclidean Geometry. 6th Edition, MAA, 1998.

[8] Schattschneider D. Coxeter and the artists: Two way inspiration. The Coxeter Legacy: Reflections and Projections, Davis C, Ellis E W (eds.), Fields Institute Comm. Ser., 46, Amer. Math. Soc, 2005, pp.258-260.

[9] Dunham D. Hyperbolic art and the poster pattern. 2003. http://www.mathaware.org/mam/03/essay1.html.

[10] Knezevic I, Sazdanovic R, Vukmirovic S. Tess package for mathematica. http://library.wolfram.com/infocenter/MathSource/4540.

[11] Hart G W. http://www.georgehart.com.

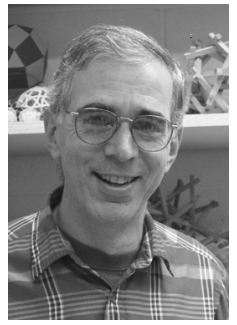

George W. Hart is a research professor in the Computer Science Department at Stony Brook University, NY. He holds his B.S. in mathematics and a Ph.D. degree in electrical engineering and computer science, both from MIT, and was previously a professor at Columbia University. He is the author of a linear algebra monograph Multidimensional Analysis (Springer Verlag, 1995) and a geometry text, Zome Geometry, (coauthored with Henri Picciotto, Key Curriculum Press, 2001). Hart is a sculptor developing innovative ways to use computer technology in the design and fabrication of his artwork. His sculpture has been displayed around the world and can be seen at http://www.georgehart.com. 